\title{
THE CUNTZ SEMIGROUP, THE ELLIOTT CONJECTURE, AND DIMENSION FUNCTIONS ON C*-ALGEBRAS
}

\author{
NATHANIAL P. BROWN, FRANCESC PERERA AND ANDREW S. TOMS
}

\begin{abstract}
We prove that the Cuntz semigroup is recovered functorially from the Elliott invariant for a large class of $\mathrm{C}^{*}$-algebras. In particular, our results apply to the largest class of simple $\mathrm{C}^{*}$-algebras for which K-theoretic classification can be hoped for. This work has three significant consequences. First, it provides new conceptual insight into Elliott's classification program, proving that the usual form of the Elliott conjecture is equivalent, among $\mathcal{Z}$-stable algebras, to a conjecture which is in general substantially weaker and for which there are no known counterexamples. Second and third, it resolves, for the class of algebras above, two conjectures of Blackadar and Handelman concerning the basic structure of dimension functions on $\mathrm{C}^{*}$-algebras. We also prove in passing that the Cuntz-Pedersen semigroup is recovered functorially from the Elliott invariant for all simple unital $\mathrm{C}^{*}$-algebras of interest.
\end{abstract}

\section{INTRODUCTION}

To any $\mathrm{C}^{*}$-algebra $A$, one can associate an invariant - the so-called Cuntz semigroup $\mathrm{W}(A)$ — which is an analogue for positive elements of the semigroup of Murray-von Neumann equivalence classes of projections $\mathrm{V}(A)$; its Grothendieck group is similarly related to the usual $\mathrm{K}_{0}$-group of $A$. (See Section 2 for definitions and details.) Cuntz's semigroup has been around for almost 30 years, but appears to be well-known to only a handful of operator algebraists. (His relation for positive elements from which $\mathrm{W}(A)$ is built, however, has received rather more attention — cf. [9], [17], [18].) There are perhaps two main reasons for this. First, classical invariants like K-theory have thus far been sufficient for most problems, especially in the context of Elliott's classification program. Second, W $(A)$ was widely regarded as either degenerate (in the infinite case) or wild and intractable (in the finite case). It is for example a monstrous uncountable semigroup

N.B. was partially supported by DMS-0554870; F.P. by the DGI MEC-FEDER through Project MTM2005-00934, and the Comissionat per Universitats i Recerca de la Generalitat de Catalunya; A.T. was supported by an NSERC Discovery Grant. 
even for Abelian $C^{*}$-algebras with contractible spectrum ([22, Lemma 5.1]), and is not continuous with respect to algebraic inductive limits.

Times change, however, and both of these rationalizations are losing legitimacy. Indeed, classical K-theory has recently shown its limitations, while $\mathrm{W}(A)$ has proven to be intimately related to the classification program (cf. [22], [27]); though it may be premature, there is even reason to hope that the Cuntz semigroup will play an important role in future classification results. With regard to the intractability issue, the second and third named authors conjectured in [16] that in spite of the discouraging situation for Abelian algebras, $\mathrm{W}(A)$ should often admit a reasonable description, and proved as much in some important cases. In the sequel we prove that this conjectural description is accurate for a class of $\mathrm{C}^{*}$-algebras large enough to encompass the vast majority of our stock-in-trade simple, separable, and nuclear $\mathrm{C}^{*}$-algebras. We then give several applications of this result.

In order to state our main result, we review briefly the description of $\mathrm{W}(A)$ discovered in [16]. Let $A$ be a unital and stably finite $\mathrm{C}^{*}$-algebra with tracial state space $\mathrm{T}(A)$, and let $\mathrm{LAff}_{b}(\mathrm{~T}(A))^{++}$denote the set of bounded, strictly positive, lower semicontinuous, and affine functions on $\mathrm{T}(A)$. Define a semigroup structure on the disjoint union

$$
\widetilde{W}(A):=V(A) \sqcup \operatorname{LAff}_{b}(\mathrm{~T}(A))^{++}
$$

as follows:

(i) if $x, y \in V(A)$, then their sum is the usual sum in $V(A)$;

(ii) if $x, y \in \operatorname{LAff}_{b}(\mathrm{~T}(A))^{++}$, then their sum is the usual (pointwise) sum in $\operatorname{LAff}_{b}(\mathrm{~T}(A))^{++}$

(iii) if $x \in V(A)$ and $y \in \operatorname{LAff}_{b}(\mathrm{~T}(A))^{++}$, then their sum is the usual (pointwise) sum of $\hat{x}$ and $y$ in $\operatorname{LAff}_{b}(\mathrm{~T}(A))^{++}$, where $\hat{x}(\tau)=\tau(x)$, $\forall \tau \in \mathrm{T}(A)$.

Equip $\widetilde{W}(A)$ with the partial order $\leq$ which restricts to the usual partial order on each of $V(A)$ and $\mathrm{LAff}_{b}(\mathrm{~T}(A))^{++}$, and which satisfies the following conditions for $x \in V(A)$ and $y \in \operatorname{LAff}_{b}(\mathrm{~T}(A))^{++}$:

(i) $x \leq y$ if and only if $\hat{x}(\tau)<y(\tau), \forall \tau \in \mathrm{T}(A)$;

(ii) $y \leq x$ if and only if $y(\tau) \leq \hat{x}(\tau), \forall \tau \in \mathrm{T}(A)$.

We can now state our main result:

Theorem. Let $A$ be a simple, unital, exact, and finite $C^{*}$-algebra which absorbs the Jiang-Su algebra $\mathcal{Z}$ tensorially. Then, there is an order isomorphism

$$
\phi: \mathrm{W}(A) \rightarrow \widetilde{\mathrm{W}}(A) .
$$


In particular, $\mathrm{W}(A)$ can be functorially reconstructed from the Elliott invariant (since $\widetilde{\mathrm{W}}(A)$ has this property).

The class of $C^{*}$-algebras to which this theorem applies already contains the largest class of $C^{*}$-algeras for which Elliott's classification conjecture can hold. It may be that the hypotheses of the theorem can be (formally) weakened by replacing $\mathcal{Z}$-stability with stable rank one and strict comparison of positive elements (see Section 2 for the definition of this last condition); we prove as much in the AH case and fully expect the ASH case to be similar (cf. Theorem 5.4).

Aside from giving a canonical presentation of $\mathrm{W}(A)$, our work has several applications which we now describe.

Elliott's Classification Program. The Elliott invariant for a unital, exact, and stably finite $C^{*}$-algebra $A$ is the 4-tuple

$$
\operatorname{Ell}(A):=\left(\left(\mathrm{K}_{0}(A), \mathrm{K}_{0}(A)^{+},\left[1_{A}\right]\right), \mathrm{K}_{1}(A), \mathrm{T}(A), r_{A}\right),
$$

where $r_{A}: \mathrm{K}_{0}(A) \times \mathrm{T}(A) \rightarrow \mathbb{R}$ is given by evaluating a trace at a $\mathrm{K}_{0}$-class. We recall two statements: the first is the Elliott conjecture as formulated in Elliott's 1994 ICM address, and the second is a seemingly weaker statement first considered in [16]. We let $\langle\bullet\rangle$ denote the Cuntz equivalence class of a positive element.

1.1 (EC). Let $A$ and $B$ be simple, separable, unital, and nuclear $C^{*}$-algebras. If there is an isomorphism

$$
\phi: \operatorname{Ell}(A) \rightarrow \operatorname{Ell}(B),
$$

then there is a $*$-isomorphism $\Phi: A \rightarrow B$ which induces $\phi$.

1.2 (WEC). Let $A$ and $B$ be simple, separable, unital, and nuclear $C^{*}$ algebras. If there is an isomorphism

$$
\phi:\left(W(A),\left\langle 1_{A}\right\rangle, \operatorname{Ell}(A)\right) \rightarrow\left(W(B),\left\langle 1_{B}\right\rangle, \operatorname{Ell}(B)\right),
$$

then there is a $*$-isomorphism $\Phi: A \rightarrow B$ which induces $\phi$.

(EC) is known to hold in great generality, particularly in the real rank zero case, but there are counterexamples, too. In fact, there are counterexamples where $A$ and $B$ have the same Elliott invariant, stable and real rank equal to one, and are even Morita and shape equivalent; they are distinguished by their Cuntz semigroups ([22, Theorem 1.1], [23]). Supporters of the classification program are thus tempted to throw $\mathrm{W}(A)$ into the invariant, i.e., to formulate the 'weak' Elliott conjecture (WEC), for which there are no known counterexamples. Critics, on the other hand, rightly point out that the addition of the seemingly intractable invariant $\mathrm{W}(A)$ to the quite reasonable invariant $\operatorname{Ell}(A)$ amounts to cheating and severely reduces the 
real-world applicability (no oxymoron intended) of any future classification results.

Our concrete presentation of $\mathrm{W}(A)$ should satisfy the critics, and breathe new life into Elliott's classification program. It reconciles the absence of $\mathrm{W}(A)$ from the invariant in the successes of Elliott's program with the necessity of $\mathrm{W}(A)$ in general. Indeed, when Corollary 5.8 below is combined with work of the second and third named authors (cf. Theorem 4.5 in [16]), we obtain:

Theorem. For either the class of (a) simple, unital AH algebras of slow dimension growth or (b) simple, unital, nuclear, and $\mathcal{Z}$-stable algebras, (EC) is equivalent to (WEC).

In other words, for the largest class of simple $C^{*}$-algebras for which (EC) might hold, there is no need to throw the Cuntz semigroup into the Elliott invariant - it's already there, in the sense that $\mathrm{W}(A)$ can be functorially reconstructed from $\operatorname{Ell}(A)$. Put another way, when trying to confirm (EC) one may further assume isomorphism of Cuntz semigroups; given the vast amount of information that $\mathrm{W}(A)$ contains, this could be a powerful tool in future classification work. (We note that for an infinite algebra as in part (b) of the theorem, the recovery of $\mathrm{W}(A)$ from the Elliott invariant is a trivial matter; $\mathrm{W}(A)$ is degenerate in this case - see Section 3 of [16].)

While the assumption of real rank zero often makes the confirmation of (EC) possible, it is by no means necessary; confirmations of (EC) for large classes of $\mathrm{C}^{*}$-algebras of real rank one abound. The latter moreover apply to $\mathrm{AH}$ algebras with slow dimension growth, or finite $\mathcal{Z}$-stable algebras (see [26] for an exhaustive list) - algebras covered by the theorem above. But while there are good philosophical grounds for believing (EC) to be true in the real rank zero case, the success of the conjecture for algebras of real rank one has been somewhat more mysterious. Our recovery of $\mathrm{W}(A)$ from $\operatorname{Ell}(A)$ goes some way toward explaining this mystery. The invariant appearing in (WEC), itself equivalent to the Elliott invariant under the assumptions of our main theorem, contains redundant information. Among finite algebras it is equivalent to the invariant

$$
\left(\mathrm{W}(A),\left\langle 1_{A}\right\rangle, \mathrm{K}_{1}(A)\right) .
$$

Thus, modulo non-commutativity, the Elliott invariant records the structure of positive elements and unitaries. One could say that it records the rough structure of polar decompositions in $A$ by recording the Cuntz equivalence class of the positive part of an arbitrary operator in $A$, and the $\mathrm{K}_{1}$-class (assuming stable rank one) of its unitary part. Whatever the philosophical interpretation, one sees that in spite of first appearances, the Elliott invariant is extremely fine. 
Blackadar-Handelman Conjectures. Following Cuntz's introduction of dimension functions for simple $C^{*}$-algebras with unit ([5]), Blackadar and Handelman made an in-depth study of traces and dimension functions ([3]). In their paper they put forth two conjectures concerning the basic structure of dimension functions for unital $C^{*}$-algebras with a trace:

(i) The lower semicontinuous dimension functions (see Section 2 for terminology) are dense in the space of all dimension functions;

(ii) The affine space of dimension functions is a simplex.

Blackadar and Handelman proved that (i) holds for commutative $C^{*}$-algebras, but did not prove (ii) in any case. The first result concerning the latter was obtained by the second named author in [14, Corollary 4.4], where it was confirmed for the class of unital $C^{*}$-algebras with real rank zero and stable rank one. To the best of the authors' knowledge, there has been no further progress on these conjectures. In the sequel we prove these conjectures under hypotheses that are even somewhat weaker than those of our main theorem above (cf. Theorems 6.4 and 6.8). Moreover, we prove a stronger version of (ii): the space of dimension functions is a Choquet simplex. Our results apply, in particular, to several classes of $\mathcal{Z}$-stable ASH algebras (see Section 4 of [26]). The $\mathcal{Z}$-stability of these algebras can only be established using the fact that they satisfy the Elliott conjecture. Thus, our confirmation of the Blackadar-Handelman conjectures for these algebras constitutes a bona fide application of K-theoretic classification theorems.

Acknowledgement: Most of this work was carried out while the first and second authors visited the third at the University of New Brunswick. We gratefully acknowledge the support and hospitality extended by UNB during our visit.

\section{Preliminaries And Notation}

Cuntz Equivalence. From here on we make the blanket assumption that all $\mathrm{C}^{*}$-algebras are separable unless otherwise stated or obviously false.

Let $A$ be a $\mathrm{C}^{*}$-algebra, and let $\mathrm{M}_{n}(A)$ denote the $n \times n$ matrices whose entries are elements of $A$. If $A=\mathbb{C}$, then we simply write $\mathrm{M}_{n}$. Let $\mathrm{M}_{\infty}(A)$ denote the algebraic limit of the direct system $\left(\mathrm{M}_{n}(A), \phi_{n}\right)$, where $\phi_{n}$ : $\mathrm{M}_{n}(A) \rightarrow \mathrm{M}_{n+1}(A)$ is given by

$$
a \mapsto\left(\begin{array}{cc}
a & 0 \\
0 & 0
\end{array}\right) .
$$


Let $\mathrm{M}_{\infty}(A)_{+}\left(\right.$resp. $\left.\mathrm{M}_{n}(A)_{+}\right)$denote the positive elements in $\mathrm{M}_{\infty}(A)$ (resp. $\left.\mathrm{M}_{n}(A)\right)$. For positive elements $a$ and $b$ in $\mathrm{M}_{\infty}(A)$, write $a \oplus b$ to denote the element $\left(\begin{array}{ll}a & 0 \\ 0 & b\end{array}\right)$, which is also positive in $\mathrm{M}_{\infty}(A)$.

Given $a, b \in \mathrm{M}_{\infty}(A)_{+}$, we say that $a$ is Cuntz subequivalent to $b$ (written $a \precsim b)$ if there is a sequence $\left(v_{n}\right)_{n=1}^{\infty}$ of elements of $\mathrm{M}_{\infty}(A)$ such that

$$
\left\|v_{n} b v_{n}^{*}-a\right\| \stackrel{n \rightarrow \infty}{\longrightarrow} 0 .
$$

We say that $a$ and $b$ are Cuntz equivalent (written $a \sim b$ ) if $a \precsim b$ and $b \precsim a$. This relation is an equivalence relation, and we write $\langle a\rangle$ for the equivalence class of $a$. The set

$$
\mathrm{W}(A):=\mathrm{M}_{\infty}(A)_{+} / \sim
$$

becomes a positively ordered Abelian monoid when equipped with the operation

and the partial order

$$
\langle a\rangle+\langle b\rangle=\langle a \oplus b\rangle
$$

$$
\langle a\rangle \leq\langle b\rangle \Leftrightarrow a \precsim b .
$$

In the sequel, we refer to this object as the Cuntz semigroup of $A$.

Given $a$ in $\mathrm{M}_{\infty}(A)_{+}$and $\epsilon>0$, we denote by $(a-\epsilon)_{+}$the element of $C^{*}(a)$ corresponding (via the functional calculus) to the function

$$
f(t)=\max \{0, t-\epsilon\}, t \in \sigma(a) .
$$

(Here $\sigma(a)$ denotes the spectrum of $a$.)

In order to ease the notation, we will use in the sequel $A_{a}$ to denote the hereditary $\mathrm{C}^{*}$-algebra generated by a positive element $a$ in $A$, that is, $A_{a}=\overline{a A a}$. Recall that, if $A$ is a separable $\mathrm{C}^{*}$-algebra, then all hereditary algebras are of this form.

Some of our results have the assumption that $A$ has moreover stable rank one, that is, the set of invertible elements is dense in $A$. We write $\operatorname{sr}(A)=1$, as is customary, to mean that the stable rank of $A$ is one. Under this condition, Cuntz subequivalence is implemented by unitaries.

The proposition below collects some facts about Cuntz subequivalence due to Kirchberg and Rørdam.

Proposition 2.1 (Kirchberg-Rørdam ([9]), Rørdam ([17])). Let $A$ be a $C^{*}$-algebra, and $a, b \in A_{+}$.

(i) $(a-\epsilon)_{+} \precsim a$ for every $\epsilon>0$.

(ii) The following are equivalent:

(a) $a \precsim b$;

(b) for all $\epsilon>0,(a-\epsilon)_{+} \precsim b$;

(c) for all $\epsilon>0$, there exists $\delta>0$ such that $(a-\epsilon)_{+} \precsim(b-\delta)_{+}$.

(iii) If $\epsilon>0$ and $\|a-b\|<\epsilon$, then $(a-\epsilon)_{+} \precsim b$. 
(iv) If moreover $\operatorname{sr}(A)=1$, then

$$
a \precsim b \text { if and only if }
$$

for every $\epsilon>0$, there is $u$ in $U(A)$ such that $u^{*}(a-\epsilon)_{+} u \in A_{b}$.

Note that, if $A_{a} \subseteq A_{b}$ for positive elements $a$ and $b$ in $A$, we have that $a \precsim b$ (by Proposition 2.1).

Traces, Quasitraces, States and Dimension Functions. As usual, we shall denote the state space of $A$ (that is, the space of positive, unital, linear functionals) by $\mathrm{S}(A)$. The set of tracial states will be denoted by $\mathrm{T}(A)$ and $\mathrm{QT}(A)$ will be used for the space of normalised 2-quasitraces on $A$ (v. $[3$, Definition II.1.1]). Note that $\mathrm{T}(A) \subseteq \mathrm{QT}(A)$, and equality holds when $A$ is exact (see [8]).

Let $\operatorname{St}\left(\mathrm{W}(A),\left\langle 1_{A}\right\rangle\right)$ denote the set of additive and order preserving maps $s$ from $\mathrm{W}(A)$ to $\mathbb{R}^{+}$having the property that $s\left(\left\langle 1_{A}\right\rangle\right)=1$. Such maps are generally called states and in the particular case of a $\mathrm{C}^{*}$-algebra, they are termed dimension functions.

Given $\tau$ in $\mathrm{QT}(A)$, one may define a map $d_{\tau}: \mathrm{M}_{\infty}(A)_{+} \rightarrow \mathbb{R}^{+}$by

$$
d_{\tau}(a)=\lim _{n \rightarrow \infty} \tau\left(a^{1 / n}\right) .
$$

This map is lower semicontinous, and depends only on the Cuntz equivalence class of $a$. It moreover has the following properties:

(i) if $a \precsim b$, then $d_{\tau}(a) \leq d_{\tau}(b)$;

(ii) if $a$ and $b$ are mutually orthogonal, then $d_{\tau}(a+b)=d_{\tau}(a)+d_{\tau}(b)$;

(iii) $d_{\tau}\left((a-\epsilon)_{+}\right) \nearrow d_{\tau}(a)$ as $\epsilon \rightarrow 0$.

Thus, $d_{\tau}$ defines a state on $\mathrm{W}(A)$. Such states are called lower semicontinuous dimension functions, and the set of them is denoted $\operatorname{LDF}(A)$. It was proved in [3, Theorem II.4.4] that $\mathrm{QT}(A)$ is a simplex; the map from $\mathrm{QT}(A)$ to $\operatorname{LDF}(A)$ defined by $(1)$ is bijective and affine ([3, Theorem II.2.2]), but generally not continuous. We also have that $\operatorname{LDF}(A)$ is a (generally proper) face of $\operatorname{DF}(A)$, see [3, Proposition II.4.6]. If $A$ has the property that $a \precsim b$ whenever $s(a)<s(b)$ for every $s \in \operatorname{LDF}(A)$, then we say that $A$ has strict comparison of positive elements or simply strict comparison.

The Grothendieck group of $\mathrm{W}(A)$ is denoted by $\mathrm{K}_{0}^{*}(A)$. The class of an element $a$ from $\mathrm{M}_{\infty}(A)_{+}$will be denoted by $[a]$. This is a partially ordered Abelian group with positive cone $\mathrm{K}_{0}^{*}(A)^{+}=\{[a]-[b] \mid b \precsim a\}$. Observe then that $\mathrm{DF}(A)=\operatorname{St}\left(\mathrm{K}_{0}^{*}(A), \mathrm{K}_{0}^{*}(A)^{+},\left[1_{A}\right]\right)$, which is the set of group morphisms $s: \mathrm{K}_{0}^{*}(A) \rightarrow \mathbb{R}$ such that $s\left(\mathrm{~K}_{0}^{*}(A)^{+}\right) \subseteq \mathbb{R}^{+}$and $s\left(\left[1_{A}\right]\right)=1$. 


\section{Duality and Traces}

If $\mathrm{S}(A)$ is the state space of a unital $\mathrm{C}^{*}$-algebra $A$ and $X=\operatorname{span}_{\mathbb{R}} \mathrm{S}(A)$ is the $\mathbb{R}$-Banach space of self-adjoint functionals on $A$ then we have two natural dualities:

$$
X=\left(A_{\mathrm{sa}}\right)^{*} \text { and } X^{*}=A_{\mathrm{sa}}^{* *},
$$

where $A_{\mathrm{sa}}\left(\right.$ resp. $\left.A_{\mathrm{sa}}^{* *}\right)$ denotes the self-adjoint elements in $A$ (resp. in the enveloping von Neumann algebra $\left.A^{* *}\right)$. Kadison's function representation (cf. [12, Theorem 3.10.3]) is a well-known application of these two facts: If $f: \mathrm{S}(A) \rightarrow \mathbb{R}$ is a bounded affine function then there exists a unique $T \in A_{\mathrm{sa}}^{* *}$ such that $f(\varphi)=\varphi(T)$, for all $\varphi \in \mathrm{S}(A)$, and $\|T\|=\|f\|$; moreover, $f$ is continuous if and only if $T \in A_{\mathrm{sa}}$.

The purpose of this section is to prove analogous results when $\mathrm{S}(A)$ is replaced by $\mathrm{T}(A)$ (cf. Theorem 3.8 and Corollary 3.10 ).

Some Conventions and Basics. For a unital $\mathrm{C}^{*}$-algebra $A$, we always consider $\mathrm{T}(A)$ a compact topological space, endowed with the weak-* topology coming from $A^{*}$. (Hence, a "continuous" function on $\mathrm{T}(A)$ means continuous with respect to this topology.) We regard the $\mathbb{R}$-linear $\operatorname{space} \operatorname{span}_{\mathbb{R}} \mathrm{T}(A)$ as a $\mathbb{R}$-Banach space, equipped with the restriction of the norm on $A^{*}$; when thinking of $\operatorname{span}_{\mathbb{R}} \mathrm{T}(A)$ as a locally convex space with respect to the weak-* topology, we will make this point explicit.

Proposition 3.1 (Jordan Decomposition). For any unital $C^{*}$-algebra $A$ and self-adjoint functional $\varphi \in A^{*}$, there exist (unique) orthogonal central projections $P_{+}, P_{-} \in A^{* *}$ such that $\varphi_{+}(a):=\varphi\left(a P_{+}\right)$and $\varphi_{-}(a):=$ $-\varphi\left(a P_{-}\right)$are positive linear functionals, $\varphi=\varphi_{+}-\varphi_{-}$and $\|\varphi\|=\left\|\varphi_{+}\right\|+$ $\|\varphi-\|$

If $\varphi$ has the property that $\varphi\left(a^{*} a\right)=\varphi\left(a a^{*}\right)$ for all $a \in A$ and $\varphi=\varphi_{+}-\varphi_{-}$ is its Jordan decomposition then $\varphi, \varphi_{+}$and $\varphi_{-}$are all tracial functionals. Consequently,

$$
\begin{aligned}
\operatorname{span}_{\mathbb{R}} \mathrm{T}(A) & =\left\{\varphi \in A^{*}: \varphi\left(a^{*}\right)=\varphi(a)^{*} \text { and } \varphi\left(a^{*} a\right)=\varphi\left(a a^{*}\right), \forall a \in A\right\} \\
& =\left\{t_{1} \tau_{1}-t_{2} \tau_{2}: t_{i} \geq 0, \tau_{i} \in \mathrm{T}(A), i=1,2\right\} .
\end{aligned}
$$

Proof. The existence (and uniqueness) of the Jordan decomposition is well known - see [20, Theorem 4.2(ii)]. So are the statements about traces (see [6, Proposition 2.8] for a more general fact), but the proofs are elementary, so we include them.

If $\varphi\left(a^{*} a\right)=\varphi\left(a a^{*}\right)$, for all $a \in A$, then $\varphi\left(u b u^{*}\right)=\varphi\left(b^{1 / 2} u^{*} u b^{1 / 2}\right)=\varphi(b)$ for all positive $b$ and unitaries $u$. Thus, by linearity, $\varphi(u b)=\varphi\left(u^{*}(u b) u\right)=$ $\varphi(b u)$ for all $b \in A$. Since every element in $A$ is a linear combination of unitaries, it follows that $\varphi(a b)=\varphi(b a)$ for all $a, b \in A$. To prove $\varphi_{ \pm}$are 
both tracial it suffices to prove $\varphi_{+}$is tracial, so we compute:

$$
\begin{aligned}
\varphi_{+}(a b) & =\varphi\left(a b P_{+}\right) \\
& =\varphi\left(a P_{+} b\right) \\
& =\varphi\left(b a P_{+}\right) \\
& =\varphi_{+}(b a) .
\end{aligned}
$$

The set equalities are an easy consequence. Indeed, since $\mathcal{S}:=\left\{t_{1} \tau_{1}-t_{2} \tau_{2}\right.$ : $\left.t_{i} \geq 0, \tau_{i} \in \mathrm{T}(A), i=1,2\right\}$ is clearly the smallest of the sets, it suffices to show that every self-adjoint $\varphi \in A^{*}$ such that $\varphi\left(a^{*} a\right)=\varphi\left(a a^{*}\right)$ belongs to $\mathcal{S}$. But, this is the content of our argument above.

Proposition 3.2. Let $f: \mathrm{T}(A) \rightarrow V$ be an affine function into a $\mathbb{R}$-vector space $V$. Then, $f$ has a unique extension to a linear function $\tilde{f}: \operatorname{span}_{\mathbb{R}} \mathrm{T}(A) \rightarrow$ $V$. If $V$ is a topological vector space and $f$ is continuous then $\tilde{f}$ is also continuous (with respect to the weak-* topology).

Proof. Given $\varphi=t_{1} \tau_{1}-t_{2} \tau_{2} \in \operatorname{span}_{\mathbb{R}} \mathrm{T}(A)$, with $t_{i} \geq 0$ and $\tau_{i} \in \mathrm{T}(A)$, we have no choice but to define

$$
\tilde{f}(\varphi)=t_{1} f\left(\tau_{1}\right)-t_{2} f\left(\tau_{2}\right) .
$$

The only question is whether or not $\tilde{f}$ is well-defined.

So, assume that $\alpha_{1} \tau_{1}-\alpha_{2} \tau_{2}=\beta_{1} \sigma_{1}-\beta_{2} \sigma_{2}$, where the scalars $\alpha_{i}$ and $\beta_{i}$ are all positive. Then

$$
c\left(\alpha_{1} \tau_{1}+\beta_{2} \sigma_{2}\right)=c\left(\beta_{1} \sigma_{1}+\alpha_{2} \tau_{2}\right) \in \mathrm{T}(A),
$$

for some positive real number $c$. Since states map $1_{A}$ to $1, c \alpha_{1}+c \beta_{2}=$ $c \beta_{1}+c \alpha_{2}=1$ and so, by affinity of $f$,

$$
c \alpha_{1} f\left(\tau_{1}\right)+c \beta_{2} f\left(\sigma_{2}\right)=c \beta_{1} f\left(\sigma_{1}\right)+c \alpha_{2} f\left(\tau_{2}\right) .
$$

Rearranging terms and dividing by $c$, we see that $\tilde{f}$ is well-defined.

Now assume $V$ is a topological vector space and $f$ is continuous. By our standing separability assumption on $A$, the weak-* topology on $A^{*}$ is metrizable and so it suffices to show that if $\left\{\varphi_{n}\right\} \subset \operatorname{span}_{\mathbb{R}} \mathrm{T}(A)$ is a weak-* convergent sequence (as opposed to net) with limit $\varphi$, then $\tilde{f}\left(\varphi_{n}\right) \rightarrow \tilde{f}(\varphi)$. Note that the principle of uniform boundedness ensures that $\sup \left\|\varphi_{n}\right\|<\infty$. Hence when we take Jordan decompositions

$$
\varphi_{n}=t_{n}^{+} \tau_{n}^{+}-t_{n}^{-} \tau_{n}^{-},
$$

where $t_{n}^{ \pm} \geq 0, \tau_{n}^{ \pm} \in \mathrm{T}(A)$ and $\left\|\varphi_{n}\right\|=t_{n}^{+}+t_{n}^{-}$, we are guaranteed to have $\sup _{n} t_{n}^{+}<\infty$ and $\sup _{n} t_{n}^{-}<\infty$. Since $\mathrm{T}(A)$ is compact, we can pass to a 
subsequence and assume that the $t_{n}^{ \pm} \mathrm{S}$ and $\tau_{n}^{ \pm} \mathrm{S}$ converge to numbers $t^{ \pm}$and, respectively, tracial states $\tau^{ \pm}$. It follows that $\varphi=t^{+} \tau^{+}-t^{-} \tau^{-}$, and thus

$$
\tilde{f}\left(\varphi_{n}\right)=t_{n}^{+} f\left(\tau_{n}^{+}\right)-t_{n}^{-} f\left(\tau_{n}^{-}\right) \rightarrow t^{+} f\left(\tau^{+}\right)-t^{-} f\left(\tau_{n}^{-}\right)=\tilde{f}(\varphi) .
$$

Cuntz-Pedersen Equivalence. There is another notion of equivalence that one can consider in $A_{+}$, first studied by Cuntz and Pedersen in [6]. Namely, for positive elements $a, b \in A_{+}$, we write $a \sim_{C P} b$ if there exist elements $u_{n} \in A$ such that

$$
a=\sum_{n} u_{n}^{*} u_{n} \text { and } b=\sum_{n} u_{n} u_{n}^{*},
$$

where convergence is in norm. By [11, Proposition 1.1] (see also [13, Corollary 3.6]), $\sim_{C P}$ is an equivalence relation. It follows from the definition, and a change of index set, that if $a_{1} \sim_{C P} b_{1}$ and $a_{2} \sim_{C P} b_{2}$ then $a_{1}+a_{2} \sim_{C P}$ $b_{1}+b_{2}$. Thus we can define the Cuntz-Pedersen semigroup to be $A_{+}$modulo the equivalence relation $\sim_{C P}$. More generally,

$$
A_{0}=\left\{a-b: a, b \in A_{+}, a \sim_{C P} b\right\}
$$

is a $\mathbb{R}$-linear subspace of $A_{\mathrm{sa}}$. In fact, [6, Theorem 2.6] asserts that $A_{0}$ is a norm-closed subspace, and hence we can factor it out.

Definition 3.3. Define a $\mathbb{R}$-Banach space by

$$
A^{q}=A_{\mathrm{sa}} / A_{0},
$$

and let $A_{+}^{q}=q\left(A_{+}\right)$be the image of $A_{+}$under the quotient map $q: A_{\mathrm{sa}} \rightarrow$ $A^{q}$.

Since $\operatorname{span}_{\mathbb{R}} \mathrm{T}(A)$ is a weak-* closed subspace of $\left(A_{\mathrm{sa}}\right)^{*}$, it has a predual (namely, the quotient of $A_{\mathrm{sa}}$ by the pre-annihilator). More precisely:

Proposition 3.4. [6, Proposition 2.7] The dual space of $A^{q}$ is isometrically isomorphic to $\operatorname{span}_{\mathbb{R}} \mathrm{T}(A)$. Moreover, the induced weak-* topology agrees with the canonical weak-* topology (coming from $A^{*}$ ).

Proof. The adjoint of the surjection $q: A_{\mathrm{sa}} \rightarrow A^{q}$ is an isometric injection

$$
q^{*}:\left(A^{q}\right)^{*} \hookrightarrow\left(A_{\mathrm{sa}}\right)^{*} .
$$

If $\varphi \in\left(A^{q}\right)^{*}$ and $a \in A$ is arbitrary, then

$$
q^{*}(\varphi)\left(a^{*} a\right)=\varphi\left(q\left(a^{*} a\right)\right)=\varphi\left(q\left(a a^{*}\right)\right)=q^{*}(\varphi)\left(a a^{*}\right),
$$

since $a^{*} a-a a^{*} \in A_{0}$. It follows that the range of $q^{*}$ is contained in $\operatorname{span}_{\mathbb{R}} \mathrm{T}(A)$. Surjectivity is trivial since every tracial state on $A$ evidently drops to a linear functional on $A^{q}$.

The agreement of weak-* topologies is evident. 
For simple, unital $\mathrm{C}^{*}$-algebras the following description of $A_{+}^{q} \backslash\{0\}$ is useful.

Proposition 3.5. [6, Theorem 5.2 and Corollary 6.4] If $A$ is simple, unital and has at least one tracial state, then

$$
A_{+}^{q} \backslash\{0\}=\left\{x \in A^{q}: \tau(x)>0, \forall \tau \in \mathrm{T}(A)\right\} .
$$

In addition, $A_{+}^{q}$ is isomorphic, as an additive semigroup, to the CuntzPedersen semigroup $A_{+} / \sim_{C P}$.

Proof. The inclusion $\subset$ is immediate from simplicity. So, proceeding by contradiction, assume that $x \in A^{q}$ and $\tau(x)>0$ for all $\tau \in \mathrm{T}(A)$, yet $x \notin$ $A_{+}^{q} \backslash\{0\}$. According to [6, Theorem 6.3], when $A$ is simple and unital the $\mathbb{R}_{+}{ }^{-}$ cone (hence convex set) $A_{+}^{q} \backslash\{0\}$ is open. Thus the Hahn-Banach theorem provides us with $\varphi \in\left(A^{q}\right)^{*}$ such that $\varphi(y) \geq \varphi(x)$ for all $y \in A_{+}^{q} \backslash\{0\}$. Since $A_{+}^{q} \backslash\{0\}$ is a cone, it follows that $\varphi(y) \geq 0$ for all $y \in A_{+}^{q} \backslash\{0\}$. Thus $\varphi$ is a positive linear functional - i.e. a multiple of an element in $\mathrm{T}(A)$ for which $\varphi(x) \leq 0$, and this contradicts our assumption.

The last statement is immediate from [6, Theorem 5.2]: If $A$ is algebraically simple (e.g. simple and unital) and $x, y \in A_{+}$then $q(x)=q(y)$ if and only if $x \sim_{C P} y$.

Corollary 3.6. If unital $C^{*}$-algebras $A$ and $B$ have non-empty affinely homeomorphic tracial state spaces then $A^{q} \cong B^{q}$.

If $A$ and $B$ are simple then the Cuntz-Pedersen semigroups $A_{+} / \sim_{C P}$ and $B_{+} / \sim_{C P}$ are also isomorphic.

Proof. Assume that $\mathrm{T}(A)$ and $\mathrm{T}(B)$ are affinely homeomorphic (with respect to the restrictions of the weak-* topologies on $A^{*}$ and, respectively, $\left.B^{*}\right)$. Then, thanks to Propositions 3.2 and $3.4, \operatorname{span}_{\mathbb{R}} \mathrm{T}(A)$ is isomorphic to $\operatorname{span}_{\mathbb{R}} \mathrm{T}(B)$ as locally convex spaces with respect to the weak-* topologies coming from $A^{q}$ and, respectively, $B^{q}$. Thus their dual spaces - i.e. $A^{q}$ and $B^{q}$ - must be isomorphic too.

It is clear that the induced isomorphism $A^{q} \rightarrow B^{q}$ will map the set $\{x \in$ $\left.A^{q}: \tau(x)>0, \forall \tau \in \mathrm{T}(A)\right\}$ bijectively onto $\left\{y \in B^{q}: \tau(y)>0, \forall \tau \in \mathrm{T}(B)\right\}$. It follows that $A_{+}^{q}$ will get mapped bijectively onto $B_{+}^{q}$; hence Proposition 3.5 implies that $A_{+} / \sim_{C P}$ and $B_{+} / \sim_{C P}$ are isomorphic too.

Tracial Analogue of Kadison's Function Representation. With the canonical predual of $\operatorname{span}_{\mathbb{R}} \mathrm{T}(A)$ in hand, our tracial version of Kadison's function representation is within sight. We just need the dual space. This has a simple description in terms of the enveloping von Neumann algebra $A^{* *}$ (indeed, it may be known to some experts, but we are unaware of a reference). 
Lemma 3.7. Let $A$ be a unital $C^{*}$-algebra with tracial simplex $\mathrm{T}(A)$. If $Z$ denotes the center of the maximal finite summand of $A^{* *}$ then there is an isometric identification

$$
Z_{\mathrm{sa}}=\left(\operatorname{span}_{\mathbb{R}} \mathrm{T}(A)\right)^{*} .
$$

In other words, the predual of $Z$ is equal to $\operatorname{span}_{\mathbb{C}} \mathrm{T}(A)$.

Proof. For convenience, let $M$ denote the maximal finite summand of $A^{* *}$ (hence $A^{* *}=M \oplus N$, with $N$ infinite). Let $\Phi: M \rightarrow Z$ be the canonical center-valued trace (cf. [19, Theorem 2.4.6]); that is, $\Phi$ is a $\sigma$-weakly continuous faithful conditional expectation onto $Z$ with the property that $\Phi(x y)=\Phi(y x)$, for all $x, y \in M$, and $\tau \circ \Phi=\tau$ for every tracial state on $M$. Though a slight abuse of notation, we will let $\Phi(a) \in Z, a \in A$, denote the composition of the maps

$$
A \hookrightarrow A^{* *} \rightarrow M \stackrel{\Phi}{\rightarrow} Z,
$$

where $A^{* *} \rightarrow M$ is the canonical quotient map.

For each $\tau \in \mathrm{T}(A)$ we use the same symbol to denote the normal extension to $A^{* *}$. Note that each such $\tau$ is supported on $M$ - i.e. $\left.\tau\right|_{N}=0$, by maximality of $M$ - and thus we have a natural inclusion $\operatorname{span}_{\mathbb{C}} \mathrm{T}(A) \subset$ $M_{*}$. Since $Z$ is a subalgebra of $M$, we have a (linear) restriction map $\operatorname{span}_{\mathbb{C}} \mathrm{T}(A) \rightarrow Z_{*}$. It is evidently isometric (hence injective) since

$$
\begin{aligned}
\left\|\left.\tau\right|_{Z}\right\|_{Z_{*}} \leq\|\tau\|_{\left(A^{* *}\right)_{*}} & =\|\tau\|_{A^{*}} \\
& =\sup \{|\tau(a)|: a \in A,\|a\| \leq 1\} \\
& =\sup \{|\tau(\Phi(a))|: a \in A,\|a\| \leq 1\} \\
& \leq\left\|\left.\tau\right|_{Z}\right\|_{Z_{*}} .
\end{aligned}
$$

To prove surjectivity of the restriction map $\operatorname{span}_{\mathbb{C}} \mathrm{T}(A) \rightarrow Z_{*}$, it suffices to show that every normal state on $Z$ is the restriction of some tracial state. So, fix a normal state $\varphi \in Z_{*}$ and define a trace $\tau$ on $A$ by $\tau(a)=\varphi(\Phi(a))$. (This is tracial since $\Phi$ is.) One easily checks that (the normal extension of) $\tau$ restricts to $\varphi$, using the fact that $\Phi$ is a $\sigma$-weakly continuous conditional expectation. This establishes the canonical isometric identification $Z_{*} \cong$ $\operatorname{span}_{\mathbb{C}} \mathrm{T}(A)$.

It follows that $Z_{\mathrm{sa}}=\left(\operatorname{span}_{\mathbb{R}} \mathrm{T}(A)\right)^{*}$, because $Z_{\mathrm{sa}}$ can be identified with the dual of the self-adjoint, normal functionals on $Z$ - i.e. the dual of $\operatorname{span}_{\mathbb{R}} \mathrm{T}(A)$.

Summarizing our duality results, we have:

Theorem 3.8. Let $A$ be a unital $C^{*}$-algebra with tracial simplex $\mathrm{T}(A)$. Then

$$
\operatorname{span}_{\mathbb{R}} \mathrm{T}(A)=\left(A^{q}\right)^{*} \text { and }\left(\operatorname{span}_{\mathbb{R}} \mathrm{T}(A)\right)^{*}=Z_{\mathrm{sa}},
$$


where $Z$ denotes the center of the maximal finite summand of $A^{* *}$.

Definition 3.9. For a unital $C^{*}$-algebra $A$, let $\mathrm{Aff}_{b}(\mathrm{~T}(A))$ denote the set of $\mathbb{R}$-valued bounded affine functions on $\mathrm{T}(A)$. Let $A_{\mathrm{sa}}^{* *} \rightarrow \mathrm{Aff}_{b}(\mathrm{~T}(A))$ be the restriction of Kadison's function representation to the tracial state space: $a \mapsto \hat{a}$, where

$$
\hat{a}(\tau)=\tau(a)
$$

for all $a \in A_{\mathrm{sa}}^{* *}$ and $\tau \in \mathrm{T}(A)$.

We assume below that $A$ is a unital $\mathrm{C}^{*}$-algebra with at least one tracial state.

Corollary 3.10. The mapping $a \mapsto \hat{a}$ gives a linear, order preserving, isometric identification of $Z_{\mathrm{sa}}$ with $\mathrm{Aff}_{b}(\mathrm{~T}(A))$. Moreover, for every continuous $f \in \operatorname{Aff}_{b}(\mathrm{~T}(A))$ there exists $a \in A_{\text {sa }}$ such that $f(\tau)=\tau(a)$, for all $\tau \in \mathrm{T}(A)$; if $A$ is simple and $f(\tau)>0$, for all $\tau \in \mathrm{T}(A)$, then we can find a positive $a \in A_{+}$with $f(\tau)=\tau(a)$.

Proof. Since $\mathrm{T}(A)$ is identified with the normal states on $Z$, the mapping $a \mapsto \hat{a} \in \operatorname{Aff}_{b}(\mathrm{~T}(A))$ is easily seen to be an order preserving, isometric injection of $Z_{\mathrm{sa}}$ into $\operatorname{Aff}_{b}(\mathrm{~T}(A))$. (Or, it follows from Kadison's function representation, applied to $Z$, and the fact that $\mathrm{T}(A)$ is dense in the set of all states on $Z$.) Surjectivity follows easily from Proposition 3.2 and Lemma 3.7 .

Similarly, if $f \in \mathrm{Aff}_{b}(\mathrm{~T}(A))$ is continuous, then Proposition 3.2 says we can extend it to a weak-* continuous linear functional $\tilde{f}$ on $\operatorname{span}_{\mathbb{R}} \mathrm{T}(A)$. Since the dual of $\operatorname{span}_{\mathbb{R}} \mathrm{T}(A)$ with respect to this topology is $A^{q}$, and $A^{q}$ is a quotient of $A_{\mathrm{sa}}$, we simply identify $\tilde{f}$ with an element in $A^{q}$ and lift it to $A_{\text {sa. }}$.

When $A$ is simple, every $x \in A^{q}$ with the property that $\tau(x)>0$, for all $\tau \in \mathrm{T}(A)$, can be lifted to a positive element thanks to Proposition 3.5. This implies the last statement, so the proof is complete.

\section{Suprema in the Cuntz Semigroup}

In this section we prove that for $\mathrm{C}^{*}$-algebras with stable rank one, the Cuntz semigroup admits suprema of countable bounded sequences in a sense that we now proceed to define.

Definition 4.1. Let $(M, \leq)$ be a preordered Abelian semigroup with identity element 0 . We say that an element $x$ in $M$ is the supremum of an increasing sequence $\left(x_{n}\right)$ of elements in $M$ provided that $x_{n} \leq x$ for each $n$ and is the smallest such $x$, meaning that if $y \in M$ and $x_{n} \leq y$ for all $n$, then necessarily $x \leq y$. 
Existence of suprema in the Cuntz semigroup was first observed by the second author in [14, Lemma 3.2] for $\mathrm{C}^{*}$-algebras with real rank zero and stable rank one. In this section we drop the condition of real rank zero and obtain the same result, albeit with considerably more effort. We have been informed by George Elliott that suprema in the Cuntz semigroup exist in full generality, a result he has proved with K. Coward and C. Ivanescu. No preprint was available at the time of writing, but we state for the record their result predates ours. It is not clear whether their result will suffice for our application, as we require a particular description of suprema in the Cuntz semigroup.

Lemma 4.2. Let $A$ be a unital and separable $C^{*}$-algebra, and let $a_{n}$ be a sequence of positive elements in $A$ such that $A_{a_{1}} \subseteq A_{a_{2}} \subseteq \cdots$. Let $A_{\infty}=\overline{\cup_{n=1}^{\infty} A_{a_{n}}}$, and let $a_{\infty}$ be a strictly positive element of $A_{\infty}$. Then

$$
\left\langle a_{\infty}\right\rangle=\sup _{n}\left\langle a_{n}\right\rangle \text {. }
$$

Moreover, for any trace $\tau$ in $\mathrm{T}(A)$, we have $d_{\tau}\left(a_{\infty}\right)=\sup _{n} d_{\tau}\left(a_{n}\right)$.

Proof. To prove that $\left\langle a_{\infty}\right\rangle \geq\left\langle a_{n}\right\rangle$, it suffices to prove that, as observed above, $A_{\infty}=A_{a_{\infty}}$. For this, it is enough to show that $A_{\infty}$ is hereditary. Indeed, if $a \in A$ and $c_{1}, c_{2} \in A_{\infty}$, then choose sequences $x_{n}$ and $y_{n}$ in $A_{a_{n}}$ such that

$$
\left\|x_{n}-c_{1}\right\| \rightarrow 0 \text { and }\left\|y_{n}-c_{2}\right\| \rightarrow 0 .
$$

Then $x_{n} a y_{n} \in A_{n}$, and since $c_{1} a c_{2}=\lim _{n} x_{n} a y_{n}$, we see that $c_{1} a c_{2}$. (Recall from, e.g. [10, Theorem 3.2.2], that a $\mathrm{C}^{*}$-subalgebra $C$ of $A$ is hereditary if and only if $c_{1} a c_{2} \in C$ whenever $a \in A$ and $c_{1}, c_{2} \in C$.)

Now assume that $\left\langle a_{n}\right\rangle \leq\langle b\rangle$ for all $n$ in $\mathbb{N}$. Choose positive elements $x_{n}$ in $A_{a_{n}}$ such that $\left\|x_{n}-a_{\infty}\right\|<\delta_{n}$, where $\delta_{n} \rightarrow 0$. It then follows by [9, Lemma 2.5 (ii)] that $\left\langle\left(a_{\infty}-\delta_{n}\right)_{+}\right\rangle \leq\left\langle x_{n}\right\rangle \leq\left\langle a_{n}\right\rangle \leq\langle b\rangle$. Thus [9, Proposition 2.6] (or [17, Proposition 2.4]) entails $\left\langle a_{\infty}\right\rangle \leq\langle b\rangle$, as desired.

Also, since $\left\langle x_{n}\right\rangle \leq\left\langle a_{n}\right\rangle \leq\left\langle a_{n+1}\right\rangle \leq\left\langle a_{\infty}\right\rangle$ for all $n$ and $\lim _{n} x_{n}=a_{\infty}$, we have that, if $\tau \in \mathrm{T}(A)$,

$$
\sup _{n \rightarrow \infty} d_{\tau}\left(a_{n}\right) \leq d_{\tau}\left(a_{\infty}\right) \leq \liminf _{n \rightarrow \infty} d_{\tau}\left(x_{n}\right) \leq \liminf _{n \rightarrow \infty} d_{\tau}\left(a_{n}\right)=\sup _{n \rightarrow \infty} d_{\tau}\left(a_{n}\right)
$$

We shall assume in the results below that $\operatorname{sr}(A)=1$. Recall that, under this assumption, Cuntz subequivalence is implemented by unitaries (by condition (iv) in Proposition 2.1). Note that, in this case, $a \precsim b$ implies that for each $\epsilon>0$, there is $u$ in $U(A)$ such that $A_{(a-\epsilon)_{+}} \subseteq u A_{b} u^{*}$. Indeed, if $a \in A_{(a-\epsilon)_{+}}$, then find a sequence $\left(z_{n}\right)$ in $A$ such that $a=$ 
$\lim _{n}(a-\epsilon)_{+} z_{n}(a-\epsilon)_{+}$. Writing $(a-\epsilon)_{+}=u c_{\epsilon} u^{*}$, with $c_{\epsilon}$ in $A_{b}$, we see that $a=u\left(\lim _{n} c_{\epsilon} u^{*} z_{n} u c_{\epsilon}\right) u^{*} \in u A_{b} u^{*}$.

Lemma 4.3. Let $A$ be a unital and separable $C^{*}$-algebra with $\operatorname{sr}(A)=1$. Let $\left(a_{n}\right)$ be a sequence of elements in $A$ such that $\left\langle a_{1}\right\rangle \leq\left\langle a_{2}\right\rangle \leq \ldots$. Then $\sup _{n}\left\langle a_{n}\right\rangle$ exists in $\mathrm{W}(A)$ and for any $\tau$ in $\mathrm{T}(A)$, we have $d_{\tau}\left(\sup _{n}\left\langle a_{n}\right\rangle\right)=$ $\sup _{n} d_{\tau}\left(a_{n}\right)$.

Proof. Define numbers $\epsilon_{n}>0$ recursively. Let $\epsilon_{1}=1 / 2$, and choose $\epsilon_{n}<$ $1 / n$ such that

$$
\left(a_{j}-\epsilon_{j} / k\right)_{+} \precsim\left(a_{n}-\epsilon_{n}\right)_{+}
$$

for all $1 \leq j<n$ and $1 \leq k \leq n$. (This is possible using [9, Proposition 2.6] and because $a_{j} \precsim a_{n}$ for $1 \leq j<n$. Notice also that $\left(a_{n}-\epsilon\right)_{+} \leq\left(a_{n}-\delta\right)_{+}$ whenever $\delta \leq \epsilon$.)

Since $\left(a_{1}-\epsilon_{1} / 2\right)_{+} \precsim\left(a_{2}-\epsilon_{2}\right)_{+}$and $\operatorname{sr}(A)=1$, there is a unitary $u_{1}$ such that

$$
A_{\left.\left(a-\epsilon_{1} / 2\right)_{+}-\epsilon_{1} / 2\right)_{+}} \subseteq u_{1} A_{\left(a_{2}-\epsilon_{2}\right)_{+}} u_{1}^{*} .
$$

But $\left.\left(a-\epsilon_{1} / 2\right)_{+}-\epsilon_{1} / 2\right)_{+}=\left(a_{1}-\epsilon_{1}\right)_{+}($see $[9$, Lemma 2.5]), so

$$
A_{\left(a-\epsilon_{1}\right)_{+}} \subseteq u_{1} A_{\left(a_{2}-\epsilon_{2}\right)_{+}} u_{1}^{*} .
$$

Continue in this way, and find unitaries $u_{n}$ in $A$ such that

$$
\begin{gathered}
A_{\left(a-\epsilon_{1}\right)_{+}} \subseteq u_{1} A_{\left(a_{2}-\epsilon_{2}\right)_{+}} u_{1}^{*} \subseteq \\
\subseteq u_{1} u_{2} A_{\left(a_{3}-\epsilon_{3}\right)_{+}} u_{2}^{*} u_{1}^{*} \subseteq \cdots \subseteq\left(\prod_{i=1}^{n-1} u_{i}\right) A_{\left(a_{n}-\epsilon_{n}\right)_{+}}\left(\prod_{i=1}^{n-1} u_{i}\right)^{*} \subseteq \cdots
\end{gathered}
$$

Use Lemma 4.2 to find a positive element $a_{\infty}$ in $A$ such that

$$
\left\langle a_{\infty}\right\rangle=\sup _{n}\left\langle\left(a-\epsilon_{n}\right)_{+}\right\rangle,
$$

and also $d_{\tau}\left(a_{\infty}\right)=\sup _{n} d_{\tau}\left(\left(a-\epsilon_{n}\right)_{+}\right) \leq \sup _{n} d_{\tau}\left(a_{n}\right)$ for any $\tau$ in $\mathrm{T}(A)$.

We claim that $\left\langle a_{\infty}\right\rangle=\sup _{n}\left\langle a_{n}\right\rangle$ as well. From this it will readily follow that $d_{\tau}\left(a_{\infty}\right)=\sup _{n} d_{\tau}\left(a_{n}\right)$.

To see that $\left\langle a_{n}\right\rangle \leq\left\langle a_{\infty}\right\rangle$ for all $n$ in $\mathbb{N}$, fix $n<m$ and recall that, by construction,

$$
\left.\left\langle\left(a_{n}-\epsilon_{n} /(m-1)\right)_{+}\right\rangle \leq\left\langle\left(a_{m}-\epsilon_{m}\right)_{+}\right)\right\rangle \leq\left\langle a_{\infty}\right\rangle .
$$

Hence, letting $m \rightarrow \infty$, we see that $\left\langle\left(a_{n}-\epsilon\right)_{+}\right\rangle \leq\left\langle a_{\infty}\right\rangle$ for any $\epsilon>0$, and so $\left\langle a_{n}\right\rangle \leq\left\langle a_{\infty}\right\rangle$ for all $n$. Conversely, if $\left\langle a_{n}\right\rangle \leq\langle b\rangle$ for all $n$ in $\mathbb{N}$, then also $\left\langle\left(a_{n}-\epsilon_{n}\right)_{+}\right\rangle \leq\langle b\rangle$ for all natural numbers $n$, and hence $\left\langle a_{\infty}\right\rangle \leq\langle b\rangle$.

Theorem 4.4. Let $A$ be a unital and separable $C^{*}$-algebra with stable rank one. Then every bounded sequence $\left\{\left\langle a_{n}\right\rangle\right\}$ in $\mathrm{W}(A)$ has a supremum $\left\langle a_{\infty}\right\rangle$ and $d_{\tau}\left(a_{\infty}\right)=\sup _{n} d_{\tau}\left(a_{n}\right)$ for any $\tau$ in $\mathrm{T}(A)$. 
Proof. Let $\left\langle x_{1}\right\rangle \leq\left\langle x_{2}\right\rangle \leq \cdots$ be given, and assume that $\left\langle x_{n}\right\rangle \leq k\left\langle 1_{A}\right\rangle$ for all $n$.

Inspection of the proof of Lemma 4.3 reveals that we may choose a sequence $\epsilon_{n}>0$ with the following properties:

(i) $\left\langle\left(x_{n}-\epsilon_{n}\right)_{+}\right\rangle \leq\left\langle\left(x_{n+1}-\epsilon_{n+1}\right)_{+}\right\rangle$.

(ii) If $\left\langle\left(x_{n}-\epsilon_{n}\right)_{+}\right\rangle \leq\langle b\rangle$ for all $n$, then $\left\langle x_{n}\right\rangle \leq\langle b\rangle$ for all $n$.

Since $\left\langle x_{n}\right\rangle \leq k\left\langle 1_{A}\right\rangle$, find $y_{n}$ in $M_{\infty}(A)_{+}$such that

$$
\left(x_{n}-\epsilon_{n}\right)_{+}=y_{n}\left(1_{A} \otimes 1_{M_{k}}\right) y_{n}^{*} .
$$

Define $a_{n}=\left(1_{A} \otimes 1_{M_{k}}\right) y_{n}^{*} y_{n}\left(1_{A} \otimes 1_{M_{k}}\right)$, which is an element of $M_{k}(A)$. Then $\left\langle a_{n}\right\rangle=\left\langle\left(x_{n}-\epsilon_{n}\right)_{+}\right\rangle \leq\left\langle a_{n+1}\right\rangle$ for all $n$. Since $M_{k}(A)$ also has stable rank one, we may use Lemma 4.3 to conclude that $\left\{\left\langle a_{n}\right\rangle\right\}$ has a supremum $\left\langle a_{\infty}\right\rangle$ with $a_{\infty}$ in $M_{k}(A)$. It follows that then $\left\langle a_{\infty}\right\rangle$ is the supremum of $\left\{\left\langle\left(x_{n}-\epsilon_{n}\right)_{+}\right\rangle\right\}$in $\mathrm{W}(A)$. Evidently, our selection of the sequence $\epsilon_{n}>0$ yields that $\left\langle a_{\infty}\right\rangle=\sup _{n}\left\langle x_{n}\right\rangle$.

The proof that $d_{\tau}\left(a_{\infty}\right)=\sup _{n} d_{\tau}\left(a_{n}\right)$ is identical to the one in Lemma 4.3.

Recall that a state $s$ on a preordered monoid $M$ with order unit $u$ is $\sigma$-normal if whenever $\left(a_{n}\right)$ is an increasing sequence and $\sup _{n} a_{n}=a$ exists, then $s(a)=\sup _{n} s\left(a_{n}\right)$. Denote the set of $\sigma$-normal states on $M$ by $\mathrm{St}_{\sigma}(M, u)$.

Corollary 4.5. Let $A$ be a unital, separable and exact $C^{*}$-algebra with stable rank one. Then $\operatorname{LDF}(A)=\mathrm{St}_{\sigma}\left(\mathrm{W}(A),\left\langle 1_{A}\right\rangle\right)$.

Proof. The inclusion $\mathrm{St}_{\sigma}\left(\mathrm{W}(A),\left\langle 1_{A}\right\rangle\right) \subseteq \operatorname{LDF}(A)$ always holds, as shown in [14, Proposition 3.3]. The converse inclusion follows directly from Theorem 4.4 and the fact that every lower semicontinuous function comes from a trace (see [3]).

Corollary 4.6. Let $A$ be a unital and separable $C^{*}$-algebra with stable rank one. If $x \in \mathrm{W}(A)$ is such that $x \leq\left\langle 1_{A}\right\rangle$, then there is a in $A$ such that $x=\langle a\rangle$.

Proof. There are a natural number $n$ and an element $b$ in $M_{n}(A)_{+}$such that $x=\langle b\rangle$. For any $m$ in $\mathbb{N}$, find elements $x_{m}$ such that

$$
(b-1 / m)_{+}=x_{m} 1_{A} x_{m}^{*},
$$

so $a_{m}:=1_{A} x_{m}^{*} x_{m} 1_{A} \in A$ and $a_{m} \sim(b-1 / m)_{+}$. Moreover, the sequence $\left\langle a_{m}\right\rangle$ is increasing, and the proof of Lemma 4.3 ensures that it has a supremum $a$ in $A$. Clearly,

$$
\langle a\rangle=\sup _{m}\left\langle a_{m}\right\rangle=\sup _{m}\left\langle(b-1 / m)_{+}\right\rangle=\langle b\rangle .
$$


Corollary 4.7. Let $A$ be a unital and separable $C^{*}$-algebra with stable rank one. If $\left\langle a_{n}\right\rangle$ is a bounded and increasing sequence of elements in $W(A)$ with supremum $\langle a\rangle$. Then $\langle a\rangle=\langle p\rangle$ for a projection $p$, if and only if, there exists $n_{0}$ such that $\left\langle a_{n}\right\rangle=\langle p\rangle$ whenever $n \geq n_{0}$.

Proof. Suppose that $\langle a\rangle=\sup _{n}\left\langle a_{n}\right\rangle=\langle p\rangle$ for a projection $p$. We may assume that all the elements $a, a_{n}$ and $p$ belong to $A$. For any $n$, we have that $a_{n} \precsim p$. On the other hand, the proof of Lemma 4.3 shows that $p=\lim _{n} b_{n}$, for some elements $b_{n} \precsim\left(a_{n}-\epsilon_{n}\right)_{+}$(where $\epsilon_{n}>0$ is a sequence converging to zero). From this it follows that for sufficiently large $n, p \precsim b_{n} \precsim\left(a_{n}-\epsilon_{n}\right)_{+} \precsim a_{n}$. Thus $p \sim a_{n}$ if $n$ is large enough, as desired.

$$
\text { 5. Surjectivity of } \iota: \mathrm{W}(A)_{+} \rightarrow \operatorname{LAff}_{\mathrm{b}}(\mathrm{T}(A))^{++}
$$

In the introduction we associated a partially ordered semigroup

$$
\widetilde{\mathrm{W}}(A)=\mathrm{V}(A) \sqcup \operatorname{LAff}_{\mathrm{b}}(\mathrm{T}(A))^{++}
$$

to a $\mathrm{C}^{*}$-algebra $A$. In order to state the results of this section and the next, we must recall the definition of the canonical map $\phi: \mathrm{W}(A) \rightarrow \widetilde{\mathrm{W}}(A)$. Our notation and terminology are drawn mostly from [16].

Let $A$ be a simple, unital, and stably finite $C^{*}$-algebra. We denote by $A_{++}$those elements of $A_{+}$which are not Cuntz equivalent to a projection in $\mathrm{M}_{\infty}(A)$, and set

$$
W(A)_{+}=\left\{\langle a\rangle \in W(A) \mid a \in \mathrm{M}_{\infty}(A)_{++}\right\} .
$$

The elements of $A_{++}$are called purely positive. Note that if $A$ has stable rank one, then $W(A)$ is the disjoint union of $V(A)$ (identified with its image into $W(A)$ via the natural map $[p] \mapsto\langle p\rangle)$, and $W(A)_{+}$. As noticed in [16, Corollary 2.9], if $A$ is either simple and stably finite or of stable rank one, we have that $W(A)_{+}$is actually a subsemigroup of $W(A)$, and is absorbing in the sense that $a+b \in W(A)_{+}$whenever $a \in V(A)$ and $b \in W(A)_{+}$.

There is a canonical map (if $A$ is simple):

$$
\iota: W(A)_{+} \rightarrow \operatorname{LAff}_{b}(\mathrm{~T}(A))^{++}
$$

given by

$$
\iota(x)(\tau)=d_{\tau}(x) .
$$

If $A$ is exact and has strict comparison, then $\iota$ is an order embedding on $W(A)_{+}\left(\left[16\right.\right.$, Proposition 3.3]). Let $\phi: \mathrm{W}(A) \rightarrow \widetilde{\mathrm{W}}(A)$ be given by $\left.\phi\right|_{V(A)}=$ $\operatorname{id}_{V(A)}$ and $\left.\phi\right|_{W(A)_{+}}=\iota$. It is proved in [16] that $\phi$ is both everywheredefined and well-defined. 
Theorem 5.1 (P-T, [16], Theorem 4.4). Let $A$ be a simple, unital, exact, and stably finite $C^{*}$-algebra with strict comparison of positive elements. Then,

$$
\phi: W(A) \rightarrow \widetilde{W}(A)
$$

is an order embedding.

Evidently, under the hypotheses of Theorem $5.1, \phi$ is an isomorphism whenever $\iota$ is surjective. In this section we prove that if one replaces the assumption of strict comparison with the formally stronger assumption of $\mathcal{Z}$-stability, then $\iota$ is in fact always surjective. We also prove that $\phi$ is an isomorphism for simple, unital, and infinite-dimensional $\mathrm{AH}$ algebras of slow dimension growth, and so for a class of algebras where $\mathcal{Z}$-stability is not known to hold.

Our first proposition follows from Corollary 3.10. In a break with convention, we let $\mathrm{CAff}(\bullet)$ denote continuous affine functions for the remainder of the paper - this is necessary as we deal also with not-necessarily-continuous affine functions.

Proposition 5.2. Assume $A_{1} \subset A_{2} \subset \cdots \subset A$ are unital subalgebras with dense union. If $A$ is simple and $f \in \mathrm{Aff}_{b}(\mathrm{~T}(A))$ is continuous and strictly positive, then for every $\varepsilon>0$ there exists $n \in \mathbb{N}$ and $0 \leq a \in A_{n}$ such that $|f(\tau)-\tau(a)|<\varepsilon$, for all $\tau \in \mathrm{T}(A)$. (Using self-adjoint a, this holds without simplicity.)

Consequently, there exists a continuous function $g \in \mathrm{Aff}\left(\mathrm{T}\left(A_{n}\right)\right)$-namely, $\hat{a}$ - whose image under the canonical map $\operatorname{Aff}\left(\mathrm{T}\left(A_{n}\right)\right) \rightarrow \operatorname{Aff}_{b}(\mathrm{~T}(A))$ is within $\varepsilon$ of $f$.

Lemma 5.3. Let $A=p(\mathrm{C}(X) \otimes \mathcal{K}) p$ be a homogeneous $C^{*}$-algebra with $X$ a compact metric space and $\operatorname{rank}(p)=n$. Let there be given $g \in \mathrm{CAff}(\mathrm{T}(A))$ satisfying $0 \leq g \leq 1$. Then, there exists $a \in \mathrm{M}_{\infty}(A)_{+}$such that $f:=\iota(a)$ satisfies

$$
0 \leq g(\tau)-f(\tau) \leq 1 / n, \forall \tau \in \mathrm{T}(A) .
$$

Proof. For each $0 \leq i \leq n-1$ define an open set

$$
A_{i}:=\{x \in X \mid g(x)>i / n\} .
$$

Notice that $A_{i} \subseteq A_{j}$ whenever $j \leq i$. Since $X$ is metric, we can find, for each $i$, a continuous function $f_{i}: X \rightarrow[0,1]$ such that $f_{i}(x) \neq 0$ if and only if $x \in A_{i}$. Put

$$
B_{i}:=\{x \in X \mid(i+1) / n \geq g(x)>i / n\}=A_{i} \backslash\left(\cup_{j>i} A_{j}\right) .
$$

and

$$
a:=\bigoplus_{i=1}^{n-1} f_{i} \cdot q \in \mathrm{M}_{\infty}(A)_{+},
$$


where $q$ is a fixed rank one projection in some $\mathrm{M}_{n}(\mathrm{C}(X)) \subseteq \mathrm{M}_{\infty}(A)$.

The tracial simplex of $A$ is a Bauer simplex, so the lower semicontinuous affine functions on $\mathrm{T}(A)$ are in bijective correspondence with the lower semicontinuous functions on the extreme boundary $\partial_{e} \mathrm{~T}(A) \cong X$ via restriction. For each $x \in X$, the value of $f(x):=\iota(a)(x)$ is the normalised rank of $a$ at $x$. In other words,

$$
\iota(a)(x):=\frac{\left|\left\{j \geq 1 \mid x \in A_{j}\right\}\right|}{n} .
$$

If $x \in\left(X \backslash A_{0}\right) \cup B_{0}$, then $f(x)=0$, and $0 \leq(g-f)(x) \geq 1 / n$ for all such $x$. If $j \geq 1$ and $x \in B_{j}$, then $f(x)=j / n$ and $j / n<g(x) \leq(j+1) / n$, and $0 \leq(g-f)(x) \leq 1 / n$ for all such $x$. Since $f$ is lower semicontinuous, so is $f-g$. A lower semicontinuous affine function on a Bauer simplex achieves its minimum on the extreme boundary, and this minimum is at least $-1 / n$ by construction. Thus, $f-g \geq-1 / n$. By affineness, $f-g \leq 0$ on every finite convex combination of extreme traces. Every point $\tau \in \mathrm{T}(A)$ is the weak-* limit of a sequence of such combinations, so the lower semicontinuity of $f-g$ yields $f-g \leq 0$ on $\mathrm{T}(A)$.

Let $A$ be a unital $\mathrm{C}^{*}$-algebra. It is well known that $A \mapsto \operatorname{CAff}(\mathrm{T}(A))$ is a covariant functor into the category of complete order-unit spaces. If $B$ is a unital $\mathrm{C}^{*}$-algebra and $\psi: A \rightarrow B$ is a $*$-homomorphism, then let

$$
\psi^{\sharp}: \mathrm{T}(B) \rightarrow \mathrm{T}(A)
$$

denote the map induced on traces. The induced map

$$
\psi^{\bullet}: \operatorname{CAff}(\mathrm{T}(A)) \rightarrow \operatorname{CAff}(\mathrm{T}(B))
$$

is then given by

$$
\psi^{\bullet}(f)(\gamma)=f\left(\psi^{\sharp}(\gamma)\right) .
$$

Let $a \in A$ be positive, with image $\iota(a) \in \operatorname{LAff}_{b}(\mathrm{~T}(A))^{+}$. Then, $\iota(\psi(a))=$ $\psi \bullet(\iota(a))$. Indeed, for $\gamma \in \mathrm{T}(B)$ we have

$$
\begin{aligned}
\iota(\psi(a))(\gamma) & =\lim _{n \rightarrow \infty} \gamma\left(\psi(a)^{1 / n}\right) \\
& =\lim _{n \rightarrow \infty} \gamma\left(\psi\left(a^{1 / n}\right)\right) \\
& =\lim _{n \rightarrow \infty} \psi^{\sharp}(\gamma)\left(a^{1 / n}\right) \\
& =\iota(a)\left(\psi^{\sharp}(\gamma)\right) \\
& =\psi^{\bullet}(\iota(a))(\gamma)
\end{aligned}
$$

Theorem 5.4. Let $A$ be a simple, unital, separable, and infinite-dimensional AH algebra of stable rank one. If A has strict comparison of positive 
elements, then the map

$$
\iota: W(A)_{+} \rightarrow \operatorname{LAff}_{b}(\mathrm{~T}(A))^{++}
$$

is surjective.

Proof. By Theorem 4.4 and Corollary 4.7 it will suffice to find, for any $f \in \operatorname{LAff}_{b}(\mathrm{~T}(A))^{++}$, a sequence $\left(a_{i}\right)_{i=1}^{\infty}$ in $A_{+}$such that $a_{i} \precsim a_{i+1},\left\langle a_{i}\right\rangle \neq$ $\left\langle a_{i+1}\right\rangle$, and

$$
\lim _{i \rightarrow \infty} d_{\tau}\left(a_{i}\right)=f(\tau)
$$

First, use the lower semicontinuity of $f$ to find a sequence $\left(f_{i}\right)_{i=1}^{\infty}$ in CAff $(\mathrm{T}(A))^{++}$satisfying

(i) $f_{i}(\tau)<f_{i+1}(\tau)$ for every $i \in \mathbb{N}$ and $\tau \in \mathrm{T}(A)$, and

(ii) $\lim _{i \rightarrow \infty} f_{i}(\tau)=f(\tau)$ for every $\tau \in \mathrm{T}(A)$.

Since the difference $f_{i}-f_{i-1}$ is continuous and strictly positive on the compact space $\mathrm{T}(A)$, it achieves a minimum, say $\epsilon_{i}>0$.

Let $A=\lim _{i \rightarrow \infty}\left(A_{i}, \phi_{i}\right)$ be an AH decomposition for $A$, i.e.,

$$
A_{i}=\bigoplus_{j=1}^{n_{i}} p_{i, j}\left(\mathrm{C}\left(X_{i, j}\right) \otimes \mathcal{K}\right) p_{i, j}
$$

for compact connected metric spaces $X_{i, j}$ and projections $p_{i, j} \in \mathrm{C}\left(X_{i, j}\right) \otimes \mathcal{K}$. Put $A_{i, j}:=p_{i, j}\left(\mathrm{C}\left(X_{i, j}\right) \otimes \mathcal{K}\right) p_{i, j}$. By Proposition 5.2 we may assume, modulo compression of our inductive system, that $f_{i} \in \phi_{i \infty}^{\bullet}\left(\mathrm{CAff}\left(\mathrm{T}\left(A_{i}\right)\right)^{+}\right)$ for each $i \in \mathbb{N}$. Let $\tilde{f}_{i}$ be a pre-image of $f_{i}$ in $\operatorname{CAff}\left(\mathrm{T}\left(A_{i}\right)\right)^{+}$. By compressing our inductive sequence again if necessary we may, by the simplicity and nonfinite-dimensionality of $A$, assume that

$$
\frac{1}{\min _{j} \operatorname{rank}\left(p_{i, j}\right)} \ll \epsilon_{i} .
$$

Use Lemma 5.3 to find, for each $1 \leq j \leq n_{i}$, an $a_{i, j} \in \mathrm{M}_{\infty}\left(A_{i, j}\right)_{+}$such that

$$
0 \leq\left.\tilde{f}_{i}\right|_{A_{i, j}}-\iota\left(a_{i, j}\right) \leq \epsilon_{i} / 2 .
$$

Put $\tilde{a}_{i}:=\sum_{j=1}^{n_{i}} a_{i, j}$. Then,

$$
0 \leq \tilde{f}_{i}-\iota\left(\tilde{a}_{i}\right) \leq \epsilon_{i} / 2 .
$$

The inequalities above are preserved under $\phi_{i \infty}^{\bullet}$, so that with $a_{i}:=\phi_{i \infty}\left(\tilde{a}_{i}\right)$ we have

$$
0 \leq f_{i}-\iota\left(a_{i}\right) \leq \epsilon_{i} / 2 .
$$

One easily checks that $\lim _{i \rightarrow \infty} d_{\tau}\left(a_{i}\right)=f(\tau)$ for each $\tau \in \mathrm{T}(A)$. Moreoever, we have $\iota\left(\left\langle a_{i}\right\rangle\right)<\iota\left(\left\langle a_{i+1}\right\rangle\right)$, whence $\left\langle a_{i}\right\rangle \neq\left\langle a_{i+1}\right\rangle$ and $a_{i} \precsim a_{i+1}$.

Now we consider the $\mathcal{Z}$-stable case. 
Lemma 5.5. Let $X$ be a compact metric space and $f \in \mathrm{Aff}(\mathrm{T}(C(X) \otimes Z))$ be a nonnegative lower semicontinous function. Then, there exists an element $\langle a\rangle \in \mathrm{W}(C(X) \otimes Z)$ such that $\|\iota(\langle a\rangle)-f\|<\epsilon$.

Proof. Since the tracial simplex of $C(X) \otimes Z$ is affinely homeomorphic to that of $C(X)$, we are again in the situation of a Bauer simplex. We first handle the case that $f=\chi_{\mathcal{O}}$, where $\mathcal{O} \subset X$ is an open set. As before, just define $a \in C(X)$ to be any function which is positive precisely on $\mathcal{O}$ and one has $\iota(\langle a\rangle)=\chi_{\mathcal{O}}$.

We can even hit multiples of such characteristic functions. Indeed, if $0<t<1$ we can find an element $z_{t} \in \mathcal{Z}$ such that $\iota\left(a \otimes z_{t}\right)$ equals $t$ times $\chi_{\mathcal{O}}$ (cf. [16, Proposition 3.2]). This, however, completes the proof since linear combinations of such characteristic functions are uniformly dense in the lower semicontinuous functions.

Theorem 5.6. Let $A$ be any simple, unital, and exact $C^{*}$-algebra which is finite and $\mathcal{Z}$-stable. Then,

$$
\iota: \mathrm{W}(A)_{+} \rightarrow \operatorname{LAff}_{b}(\mathrm{~T}(A))^{++}
$$

is surjective.

Proof. It suffices to show that if $f \in \operatorname{LAff}_{b}(\mathrm{~T}(A))^{++}$is continuous then we can approximate it arbitrarily well by elements in $\iota\left(\mathrm{W}(A)_{+}\right)$.

By Corollary 3.10, we can find $0 \leq a \in A$ such that $f=\hat{a}$. Let $\psi$ : $\mathcal{Z} \otimes \mathcal{Z} \rightarrow \mathcal{Z}$ be any $*$-isomorphism, and define

$$
\phi: A \otimes \mathcal{Z} \otimes \mathcal{Z} \rightarrow A \otimes \mathcal{Z} \otimes \mathcal{Z}
$$

by

$$
\phi\left(a \otimes z_{1} \otimes z_{2}\right)=a \otimes \psi\left(z_{1} \otimes z_{2}\right) \otimes 1_{\mathcal{Z}} .
$$

By [25, Corollary 1.12], $\phi: A \rightarrow A$ is approximately inner, whence $\widehat{\phi(a)}=\hat{a}$. We will thus assume below that upon identifying $A$ with $A \otimes \mathcal{Z}$, we have $a \in A \otimes 1_{\mathcal{Z}}$.

Let $B=C^{*}(a) \otimes \mathcal{Z}$ and now regard $\hat{a}$ as a continuous affine function on the tracial space of $B$. By the previous lemma we can approximate $\hat{a} \in \mathrm{Aff}_{b}(\mathrm{~T}(B))$ by the image of $\mathrm{W}(B)$. By functoriality, it follows that $f$ is approximated by $\iota\left(\mathrm{W}(A)_{+}\right)$.

Remark 5.7. It is proved in [24] that a simple, unital, and infinite-dimensional AH algebra of slow dimension growth has strict comparison; such algebras also have stable rank one by the main results of [2].

Corollary 5.8. Let $A$ be a simple, unital, and finite $C^{*}$-algebra which is either exact and $\mathcal{Z}$-stable or an infinite-dimensional $A H$ algebra of slow 
dimension growth. Then,

$$
\phi: \mathrm{W}(A) \rightarrow \widetilde{\mathrm{W}}(A)
$$

is an order isomorphism

Proof. Knowing the surjectivity of $\iota$ for these two classes of algebras, the result follows from Theorem 5.1.

We conjecture that Corollary 5.8 holds for simple, separable, unital ASH algebras with strict slow dimension growth, and so, by deep results of $\mathrm{Q}$. Lin and N. C. Phillips, for a large class of $\mathrm{C}^{*}$-dynamical systems.

\section{The Blackadar-Handelman Conjectures}

We now resolve the two conjectures of Blackadar and Handelman discussed in the introduction for classes of $\mathrm{C}^{*}$-algebras more general than the one considered in our main theorem. Throughout this section $\phi: \mathrm{W}(A) \rightarrow$ $\widetilde{\mathrm{W}}(A)$ is the map defined in section 5 .

Lemma 6.1. Let $\mathcal{S} \subset \operatorname{Aff}_{b}(\mathrm{~T}(A))$ be any sub-semigroup containing the constant function 1 , endowed with the pointwise (pre)order. If $\varphi: \mathcal{S} \rightarrow \mathbb{R}$ is any state then there exists a net of traces $\left\{\tau_{\lambda}\right\}_{\lambda \in \Lambda} \subset \mathrm{T}(A)$ such that

$$
\varphi(s)=\lim _{\lambda \rightarrow \infty} s\left(\tau_{\lambda}\right),
$$

for all $s \in \mathcal{S}$.

Proof. Thanks to [4, Corollary 2.7], we may extend the state $\varphi$ to a state on all of $\operatorname{Aff}_{b}(\mathrm{~T}(A))$; i.e., we may assume $\mathcal{S}=\operatorname{Aff}_{b}(\mathrm{~T}(A))$.

However, every state on $\operatorname{Aff}_{b}(\mathrm{~T}(A))$ is actually a bounded linear functional (cf. [7, Lemma 6.7]). That is, $\varphi \in\left(\operatorname{Aff}_{b}(\mathrm{~T}(A))\right)^{*}=Z_{s a}^{*}$, by Lemma 3.7. Moreover, $\varphi$ defines a positive linear functional on $Z_{s a}^{*}$, since $\varphi(0)=0$ and $\varphi$ is order preserving. Since the normal states on $Z$ are weak- $*$ dense in the set of all states, it follows that $\varphi \in Z_{s a}^{*}$ can be approximated by a net $\left\{\tau_{\lambda}\right\}_{\lambda \in \Lambda} \subset \mathrm{T}(A)$.

The following lemma is well known.

Lemma 6.2. Every infinite-dimensional $C^{*}$-algebra contains a positive element with infinite spectrum.

Corollary 6.3. Let $A$ be a simple, unital, and infinite-dimensional $C^{*}$ algebra. Then, A contains a purely positive element.

Proof. By the previous lemma, there is a positive element $a \in A$ with infinite spectrum. Choose an accumulation point $x \in \sigma(a)$. Let $f$ be a continuous function on $\sigma(a)$ such that $f(t)$ is nonzero if and only if $t \neq x$. Then, $f(a)$ 
is positive and has zero as an accumulation point of its spectrum. $f(a)$ is thus purely positive by [16, Proposition 2.1].

Theorem 6.4. Let $A$ be a simple, unital, exact, and stably finite $C^{*}$-algebra for which

$$
\phi: \mathrm{W}(A) \rightarrow \widetilde{\mathrm{W}}(A)
$$

is an order-embedding. Then, $\operatorname{LDF}(A)$ is dense in $\operatorname{DF}(A)$.

Proof. We may assume that $A$ is infinite-dimensional, whence $\mathrm{W}(A)_{+}$is non-empty by Corollary 6.3. Thus, $\mathrm{K}_{0}^{*}(A)$ is order-isomorphic to $G\left(\mathrm{~W}(A)_{+}\right)$ (see [16, Lemma 5.5]). Let $\gamma: \mathrm{W}(A)_{+} \rightarrow G\left(\mathrm{~W}(A)_{+}\right)$denote the natural Grothendieck map.

If we pick any $c$ in $\mathrm{W}(A)_{+}$, then we can define an order-isomorphism $\alpha$ by

$$
\alpha([p])=\gamma(\langle p\rangle+c)-\gamma(c)
$$

if $p$ is a projection, and

$$
\alpha([a])=\gamma(\langle a\rangle)
$$

if $\langle a\rangle \in \mathrm{W}(A)_{+}$. We thus have that, by composition, $\mathrm{K}_{0}^{*}(A)$ is orderisomorphic to a subgroup $\mathcal{S}$ of $\left\{f-g \mid f, g \in \mathrm{LAff}_{b}(\mathrm{~T}(A))^{++}\right\}$via

$$
[a]-[b] \mapsto \widehat{a}-\widehat{b},
$$

where $\widehat{a}(\tau)=d_{\tau}(a)$ (for any $\tau$ in $\mathrm{T}(A)$ ). Note that under this orderisomorphism, [1] is mapped to $\left(1 \oplus c^{\prime}\right)^{-}-\widehat{c^{\prime}}=1+\widehat{c^{\prime}}-\widehat{c^{\prime}}=1$, where $c^{\prime}$ is any purely positive element such that $\left\langle c^{\prime}\right\rangle=c$.

Next, if $d \in \operatorname{DF}(A)$, then by the isomorphism we may think of $d$ as a normalized state on the image $\mathcal{S}$, which is a subsemigroup of $\operatorname{Aff}_{b}(\mathrm{~T}(A))$ containing the constant function 1. By Lemma 6.1, there is a net of traces $\left\{\tau_{\lambda}\right\}$ in $\mathrm{T}(A)$ such that $d(s)=\lim _{\lambda} s\left(\tau_{\lambda}\right)$ for any $s$ in $\mathcal{S}$. In particular, for $a$ in $A$ :

$$
d([a])=\lim _{\lambda}\left(\widehat{a}\left(\tau_{\lambda}\right)\right)=\lim _{\lambda} d_{\tau_{\lambda}}(a),
$$

and since $a \mapsto d_{\tau_{\lambda}}(a)$ is in $\operatorname{LDF}(A)$, the proof is complete.

Remark 6.5. The order-embedding hypothesis above is satisfied whenever $A$ has strict comparison. For example, it suffices to know $A$ is $\mathcal{Z}$-stable or an AH algebra of slow dimension growth, though this is overkill as it implies $\phi$ is an order-isomorphism.

Definition 6.6. Let $(M, \leq)$ be a preordered monoid. We say that $M$ satisfies the Riesz Interpolation Property if whenever $x_{1}, x_{2}, y_{1}, y_{2} \in M$ satisfy $x_{i} \leq y_{j}$ for all $i$ and $j$, then there is $z$ in $M$ such that $x_{i} \leq z \leq y_{j}$.

Lemma 6.7. Let $K$ be a metrizable compact convex set. Then $\operatorname{LAff}_{b}(K)^{++}$, equipped with the pointwise ordering, is an interpolation monoid. 
Proof. Let there be given functions $f_{1}, f_{2}, g_{1}, g_{2}$ in $\operatorname{LAff}_{b}(K)^{++}$such that $f_{i} \leq g_{j}$ for $i, j=1,2$.

Since $K$ is metrizable, we may write $f_{i}=\sup _{n} f_{i, n}$, where $f_{i, n} \in \operatorname{CAff}(K)^{++}$ and $f_{i, n} \leq f_{i, n+1}$ for $i=1,2$ and all $n$. There is $h_{1}$ in $\operatorname{CAff}(K)^{++}$such that $f_{i, 1} \leq h_{1} \leq g_{j}$, by, e.g. [7, ].

Next, since $f_{i, 2}, h_{1} \leq g_{j}(i, j=1,2)$, there is $h_{2}$ in CAff $(K)^{++}$such that

$$
f_{i, 2}, h_{1} \leq h_{2} \leq g_{j} .
$$

Continue in this way to find an increasing sequence $h_{n}$ in $\operatorname{CAff}(K)^{++}$ such that $f_{i, n} \leq h_{n} \leq g_{j}$ for $i, j=1,2$ and all $n$. Put $h=\sup _{n} h_{n}$, which is an element of LAff $(K)^{++}$(as it is a supremum of continuous and affine functions). Then, by construction $f_{i} \leq h \leq g_{j}$ for all $i, j$.

Theorem 6.8. Let $A$ be a simple, unital, exact, and stably finite $C^{*}$-algebra. If

$$
\phi: \mathrm{W}(A) \rightarrow \widetilde{\mathrm{W}}(A)
$$

is an order isomorphism, then $\operatorname{DF}(A)$ is a Choquet simplex.

Proof. We may assume that $A$ is infinite-dimensional - the finite-dimensional case follows from the fact that $\mathrm{V}(A) \cong \mathrm{W}(A)([24])$.

Since $A$ is infinite dimensional, the semigroup $\mathrm{W}(A)_{+}$is non-empty by Corollary 6.3. Thus, we may use [16, Lemma 5.2], which ensures that the partially ordered group $K_{0}^{*}(A)$ is order-isomorphic to $G\left(\mathrm{~W}(A)_{+}\right)$(with its natural ordering induced by the partial order in $\left.\mathrm{W}(A)_{+}\right)$. Since, as just mentioned, $\mathrm{W}(A)_{+} \cong \mathrm{LAff}_{b}(\mathrm{~T}(A))^{++}$, Lemma 6.7 applies to conclude that $\mathrm{W}(A)_{+}$is an interpolation monoid. But then we can use [14, Lemma 4.2], to see that $G\left(\mathrm{~W}(A)_{+}\right)$is an interpolation group.

Therefore, $\left(K_{0}^{*}(A), K_{0}^{*}(A)^{++}\right)$is an interpolation group and thus $\mathrm{DF}(A)$, being the state space of $K_{0}^{*}(A)$, is a Choquet simplex, by e.g. [7, Theorem 10.17].

Combining Theorems 6.4 and 6.8 with Corollary 5.8 now gives:

Corollary 6.9. Let $A$ be a simple, unital, finite $C^{*}$-algebra which is either exact and $\mathcal{Z}$-stable or $A H$ with slow dimension growth. Then $\mathrm{DF}(A)$ is a Choquet simplex and $\operatorname{LDF}(A)$ is dense in it.

\section{REFERENCES}

1. Blackadar, B.: Comparison theory for simple $C^{*}$-algebras, in Operator Algebras and Applications, eds. D. E. Evans and M. Takesaki, LMS Lecture Notes Series, 135, Cambridge Univ. Press, 1988, pp. 21-54.

2. Blackadar, B., Dădărlat, M., and Rørdam, M.: The real rank of inductive limit $C^{*}$-algebras Math. Scand. 69 (1991), pp. 211-216 
3. Blackadar, B. and Handelman, D.: Dimension functions and traces on $C^{*}$-algebras, J. Funct. Anal., 45 (1982), pp. 297-340.

4. Blackadar, B. and Rørdam, M.: Extending states on preordered semigroups and the existence of quasitraces on $C^{*}$-algebras, J. Algebra, 152 (1992), pp. 240-247.

5. Cuntz, J.: Dimension functions on simple $C^{*}$-algebras, Math. Ann., 233 (1978), pp. $145-153$.

6. Cuntz, J. and Pedersen, G.K.: Equivalence and traces on $C^{*}$-algebras, J. Funct. Anal., 33 (1979), pp. 135-164.

7. Goodearl, K. R.: Partially Ordered Abelian Groups with Interpolation, Math. Surveys and Monographs 20, Amer. Math. Soc., Providence, 1986.

8. Haagerup, U.: Quasi-traces on exact $C^{*}$-algebras are traces, preprint, 1991.

9. Kirchberg, E. Rørdam, M.: Non-simple purely infinite $C^{*}$-algebras, Amer. J. Math., 122 (2000), pp. 637-666.

10. Murphy, G.J.: $C^{*}$-algebras and operator theory, Academic Press, Inc., 1990.

11. Pedersen, G.K.: Measure theory for $C^{*}$-algebras III, Math. Scand., 25 (1969), pp. 71-93.

12. Pedersen, G.K.: $C^{*}$-algebras and their automorphism groups. London Mathematical Society Monographs, 14. Academic Press, Inc. [Harcourt Brace Jovanovich, Publishers], London-New York, 1979.

13. Pedersen, G.K.: Factorization in $C^{*}$-algebras, Expo. Math., 16 (1998), pp. 145-156.

14. Perera, F.: The structure of positive elements for $C^{*}$-algebras with real rank zero, International J. Math., 8 (1997), pp. 383-405.

15. Perera, F.: Ideal structure of multiplier algebras of simple $C^{*}$-algebras with real rank zero, Canad. J. Math., 53 (2001), pp. 592-630.

16. Perera, F. and Toms, A. S.: Recasting the Elliott Conjecture, Math. Ann., to appear, arXiv preprint math.OA/0601478 (2006).

17. Rørdam, M.: On the structure of simple $C^{*}$-algebras tensored with a UHF-algebra. II, J. Funct. Anal., 107 (1992), pp. 255-269.

18. Rørdam, M.: The stable and the real rank of $\mathcal{Z}$-absorbing $C^{*}$-algebras, International J. Math., 15 (2004), pp. 1065-1084.

19. Sakai, S.: $C^{*}$-algebras and $W^{*}$-algebras. Reprint of the 1971 edition. Classics in Mathematics. Springer-Verlag, Berlin, 1998.

20. Takesaki, M.: Theory of operator algebras. I. Reprint of the first (1979) edition. Encyclopaedia of Mathematical Sciences, 124. Operator Algebras and Non-commutative Geometry, 5. Springer-Verlag, Berlin, 2002.

21. Thomsen, K.: Inductive Limits of Interval Algebras: The Tracial State Space, Amer. J. Math. 116 (1994), 605-620

22. Toms, A. S.: On the classification problem for nuclear $C^{*}$-algebras, Ann. of Math. (2), to appear, arXiv preprint math.OA/0509103 (2005).

23. Toms, A. S.: Distinguishing non-Z-Z-stable $C^{*}$-algebras with identical K-theory, in preparation.

24. Toms, A. S.: Stability in the Cuntz semigroup of a commutative $C^{*}$-algebra, arXiv preprint math.OA/0607099 (2006)

25. Toms, A. S. and Winter, W.: Strongly self-absorbing $C^{*}$-algebras, Trans. Amer. Math. Soc., to appear, arXiv preprint math.OA/0502211

26. Toms, A. S. and Winter, W.: Z-stable ASH algebras, Canad. J. Math., to appear, arXiv preprint math.OA/0508218

27. Toms, A. S. and Winter, W.: The Elliott conjecture for Villadsen algebra of the first type, in preparation 
Department of Mathematics, Penn State University, State College, PA 16802 USA

E-mail address: nbrown@math.psu.edu

Departament de Matemàtiques, Universitat Autònoma de Barcelona, 08193 Bellaterra, Barcelona, Spain

E-mail address: perera@mat.uab.cat

Department of Mathematics and Statistics, York University, 4700 Keele St. Toronto, Ontario, M3J 1P3 Canada

E-mail address: atoms@mathstat.yorku.ca 